\title{
Skeletal Muscle Response to Exercise Training in Congestive Heart Failure
}

John R. Minotti, Emily C. Johnson, Tracee L. Hudson, Glen Zuroske, Glen Murata, Eiichi Fukushima, Thomas G. Cagle, Thomas W. Chick, Barry M. Massie, and Milton V. Icenogle

Veterans Administration Medical Center, Lovelace Medical Foundation, and the Clinical Research Center of the University of New Mexico, Albuquerque, New Mexico 87108; and the Veterans Administration Medical Center, and University of California, San Francisco, California 94121

\begin{abstract}
To examine the ability of the skeletal muscle of congestive heart failure (CHF) patients to adapt to chronic exercise, five patients performed localized nondominant wrist flexor training for $28 \mathrm{~d}$. Inorganic phosphate $\left(\mathrm{P}_{\mathrm{i}}\right)$ and phosphocreatine (PCr) were monitored by magnetic resonance spectroscopy in both forearms at rest and during submaximal wrist flexion exercise at $6,12,24$, and $36 \mathrm{~J} \cdot \mathrm{min}^{-1}$ before and after exercise training. Simultaneous measurements of limb blood flow were made by plethysmography at 12,24 , and $36 \mathrm{~J} \cdot \mathrm{min}^{-1}$. Forearm muscle mass and endurance were measured by magnetic resonance imaging and wrist flexion exercise before and after training. The $P_{i} / P C r$ ratio and $p H$ were calculated from the measured $P_{i}$ and PCr. Exercise cardiac output, heart rate, plasma norepinephrine, and lactate measured during training were not elevated above resting values, confirming that training was localized to the forearm flexor muscles. After training, muscle bioenergetics, as assessed by the slope of the regression line relating $\mathbf{P}_{\mathrm{i}} / \mathrm{PCr}$ to submaximal workloads, were improved in the trained forearm of each patient, although muscle mass, limb blood flow, and pH were unchanged. Forearm endurance increased by $>260 \%$ after training. In the dominant untrained forearm, none of the measured indices were affected. We conclude that localized forearm exercise training in CHF patients improves muscle energetics at submaximal workloads in the trained muscle, an effect which is independent of muscle mass, limb blood flow, or a central cardiovascular response during training. These findings indicate that peripheral muscle metabolic and functional abnormalities in CHF can be improved without altering cardiac performance. (J. Clin. Invest. 1990. 86:751-758.) Key words: congestive heart failure - exercise training - muscle metabolism - magnetic resonance spectroscopy
\end{abstract}

\section{Introduction}

Exercise intolerance is a frequent symptom in congestive heart failure (CHF) $)^{1}(1-3)$. Although CHF patients commonly have elevated pulmonary venous pressures and frequent complaints

Address reprint requests to Dr. John Minotti, Department of Internal Medicine, University of California, San Francisco, Section of Cardiology (III C), Veterans Administration Medical Center, 4150 Clement St., San Francisco, CA 94121.

Received for publication 14 November 1989 and in revised form 25 April 1990.

1. Abbreviations used in this paper: CHF, congestive heart failure; MRS, magnetic resonance spectroscopy; $\mathrm{P}_{\mathrm{i}}$, inorganic phosphate; $\mathrm{PCr}$, phosphocreatine.

The Journal of Clinical Investigation, Inc.

Volume 86, September 1990, 751-758 of dyspnea during exercise, muscle fatigue is the primary determinant of exercise capacity (3). Consequently, activity is limited to avoid fatigue, which undoubtedly causes deconditioning and leads to a cycle of progressively worsening exercise tolerance.

Several observations suggest that changes in the periphery are important determinants of exercise performance in CHF. First, several investigators have observed that left ventricular function and systemic hemodynamics correlate poorly with exercise capacity (2). Second, despite rapid central hemodynamic improvement with medication, parallel improvements in exercise capacity do not occur (4). Third, skeletal muscle atrophy is common in advanced heart failure (5). Finally, recent studies indicate that the skeletal muscle metabolic responses to submaximal exercise are abnormal in patients with chronic heart failure (6-9).

In a recent study (10), patients with stable CHF improved peak systemic oxygen consumption in response to 4-6 mo of systemic exercise training. After training, central hemodynamics were not significantly improved; however, peripheral changes measured in the exercising limb suggested an improved skeletal muscle response to exercise. Since the conditioning stimulus was systemic, it is unclear if the measured changes in the exercising limb are attributable solely to peripheral adaptations.

Therefore, the goals of the present study were $(a)$ to determine if skeletal muscle of CHF patients can adapt to an exercise conditioning regimen that is not accompanied by a systemic exercise response and, if so $(b)$ to determine whether there are associated changes in muscle mass, peripheral blood flow, or high-energy phosphate metabolism.

\section{Methods}

Study design (Table I). Studies were performed on subjects before and after $28 \mathrm{~d}$ of localized forearm training. The sequence of testing was as follows. On day one, patients were admitted to the Veterans Administration Medical Center in Albuquerque, resting forearm blood flow was measured by venous occlusion plethysmography, and peak systemic oxygen uptake $\left(\mathrm{VO}_{2}\right)$ was determined by cycle ergometry. On day two, forearm muscle cross-sectional area was determined by magnetic resonance imaging, ventricular function by echocardiography, and strength by quantifying maximal voluntary contraction. On day three, submaximal forearm exercise was performed in the bore of a 1.9-T NMR unit while simultaneous measurements of forearm muscle metabolism and blood flow were made. On day four, patients were transferred to the Clinical Research Center at the University of New Mexico School of Medicine and the first measurement of forearm exercise endurance was made. On days five and six, subjects rested. On day seven, subjects performed the second forearm endurance protocol. For the next $4 \mathrm{wk}$, each subject remained in the clinical research center and trained $6 \mathrm{~d}$ out of each week. The testing cycle outlined above was repeated after the 28-d training period. The protocol was approved by the Human Research Review Committee of the Veterans Administra- 
Table I. Study Design

\begin{tabular}{cl}
\hline \multicolumn{1}{c}{ Day } & \multicolumn{1}{c}{ Measurement } \\
\hline 1 and 43 & $\begin{array}{l}\text { Peak oxygen consumption } \\
\text { Resting limb blood flow } \\
\text { Magnetic resonance imaging } \\
2 \text { and } 37\end{array}$ \\
& Maximal voluntary contraction \\
& Ejection fraction \\
3 and 39 & Exercise spectroscopy \\
4 & Forearm endurance practice \\
7 and 42 & Forearm endurance test \\
$8-36$ & Training $(6 d / w k)$ \\
\hline
\end{tabular}

tion Medical Center in Albuquerque and the Institutional Review Board of the Lovelace Medical Foundation.

Subjects (Table II). Male patients with a history of chronic CHF for at least 6 mo were recruited from the Veterans Administration Medical Center in Albuquerque and gave written informed consent. The criteria for $\mathrm{CHF}$ were clinical, including a history of dyspnea on exertion, fatigue, or fluid retention, with confirmation by an echocardiographic ejection fraction of $<40 \%$, and reduced peak exercise tolerance $(<25$ $\mathrm{ml} \mathrm{O}_{2}$ per kg body wt per min) determined by incremental cycle ergometry. The etiology of CHF was idiopathic in four patients and coronary artery disease in one. Patients were excluded if they had experienced myocardial infarction within 6 mo or their exercise test was limited by chest pain rather than fatigue or dyspnea. Other criteria for exclusion included initiation of long-acting nitrates or angiotensin converting enzyme inhibitor therapy within $3 \mathrm{mo}$, hemodynamically significant valvular disease, chronic obstruction pulmonary disease, arthritis, or other mechanical limitations to cycle ergometry. Patients with peripheral vascular and neurologic disease were also excluded. Cardiac medications were not altered through the duration of the study.

Phosphorus-31 nuclear magnetic resonance spectroscopy $\left({ }^{31} P\right.$ $M R S$ ). A nuclear magnetic resonance spectrometer/imager (Nalorac, Quest 4300) with a 1.9-T, 30-cm bore superconducting magnet (Oxford Instruments) was used to collect ${ }^{31}$ P-MRS data. The subject's forearm rested over an electrically balanced 4-cm-diam, two-turn surface coil made of 14-gauge copper wire under a 3-mm-thick acrylic sheet. A copper foil on the top surface of the acrylic (except near the coil) ensured that the forearm was well grounded electrically. Guides on both sides of the forearm positioned it reproducibly in the transverse direction. The longitudinal positioning of the arm was determined by the exercise handle. Both the exercise apparatus and the surface coil were mounted rigidly in the magnet (Fig. 1).

Table II. Physical Characteristics of Subjects

\begin{tabular}{lccccc}
\hline Subject & Age & $\begin{array}{c}\text { Cardiac } \\
\text { medications }\end{array}$ & $\begin{array}{c}\text { Peak initial } \\
\mathrm{VO}_{2}\end{array}$ & $\begin{array}{c}\text { Ejection } \\
\text { fraction }\end{array}$ & $\begin{array}{c}\text { NYHA } \\
\text { class }\end{array}$ \\
\hline & $y r$ & & $\mathrm{ml} \cdot \mathrm{kg}^{-1} \cdot \mathrm{min}^{-1}$ & $\%$ & \\
S.B. & 64 & C, D, F & 11.9 & 15 & III \\
E.T. & 64 & D, F, H & 17.7 & 35 & II \\
R.R. & 59 & C, D, F & 11.4 & 30 & III \\
J.K. & 45 & C, N & 22.1 & 30 & II \\
P.V. & 67 & C, D, F & 14.3 & 25 & III \\
Mean & 60 & & 15.5 & 27 & \\
SD & \pm 9 & & \pm 4.5 & \pm 7.6 & \\
& & & & &
\end{tabular}

C, captopril; D, digoxin; F, furosemide; H, hydralazine; N, nitroglycerin; NYHA, New York Heart Association.

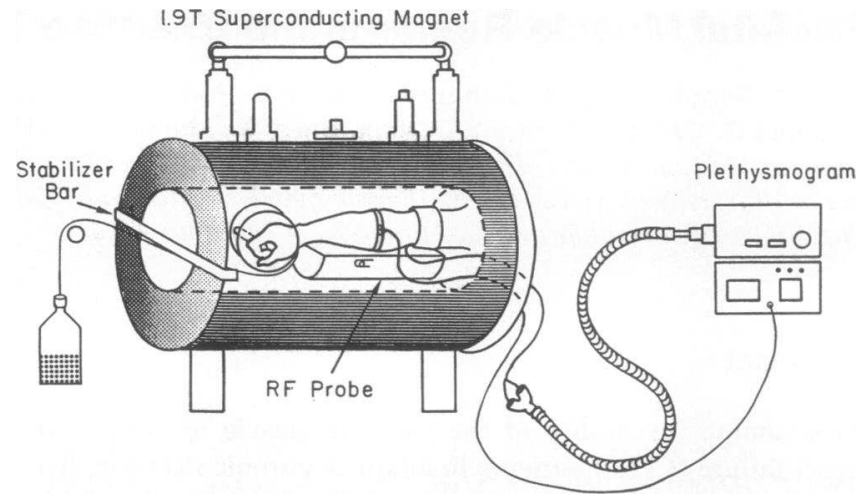

Figure 1. Exercise apparatus. Forearm muscle metabolism and blood flow were determined in the bore of a 1.9-T nuclear magnetic resonance unit at rest and during progressive exercise. Metabolism was determined by ${ }^{31} \mathrm{P}$-MRS and blood flow was measured by venous occlusion plethysmography.

The static magnetic field was made uniform by shimming on a phantom containing a standard solution of phosphoric acid with the phosphorus coil tuned to the proton frequency. The shimmed proton line width for the magnitude spectrum was $40 \mathrm{~Hz}$. Complex free induction decays were collected after $45-\mu \mathrm{S}$ pulses, which were $180^{\circ}$ pulses at the sample surface and $90^{\circ}$ pulses $12 \mathrm{~mm}$ into the sample. The full sweep width was $3 \mathrm{kHz}$, with a corresponding filter setting. After evaluating the data for steady-state metabolism, defined as a constant inorganic phosphate/phosphocreatine $\left(\mathrm{P}_{\mathrm{i}} / \mathrm{PCr}\right)$ ratio, the last 60 free induction decays $(5 \mathrm{~min})$ at rest and at each exercise load were used for analysis. The spectra were multiplied by a decaying exponential to give $7 \mathrm{~Hz}$ line broadening, then baseline corrected, Fourier transformed, and phased. Relative $\mathrm{P}_{\mathrm{i}}, \mathrm{PCr}$, and $\beta$-ATP spectral peak heights were measured from the baseline. Muscle intracellular $\mathrm{pH}$ was calculated from the chemical shift difference $(\sigma)$ in parts per million between $P_{i}$ and $\mathrm{PCr}$ :

$\mathrm{pH}=\frac{6.75+\log (\sigma-3.27)}{(5.69-\sigma)}$

The ratio of $\mathrm{P}_{\mathrm{i}} / \mathrm{PCr}$ was calculated from the spectral peak heights. ATP concentrations during exercise were calculated using the $\beta$-ATP resonance at each workload assuming a resting mean ATP concentration of $8.2 \mathrm{mM}(11)$

Exercise protocol in the magnet. Subjects arrived at the laboratory postprandial. Before exercise, each subject rested his forearm in the magnet for $\sim 15$ min during magnetic field shimming. Exercise consisted of wrist flexion over an arc (linear distance $=96 \mathrm{~mm}$ ) against a handle connected to a weight through a pulley (Fig. 1). Flexion repetition was cued every $5 \mathrm{~s}$ with a flashing light so that it would occur just after ${ }^{31}$ P-MRS data collection. A free induction decay was collected every $5 \mathrm{~s}$ during rest and exercise from the forearm flexors which were positioned over the radiofrequency coil. After a 5-min warm-up exercise at $6 \mathrm{~J} \cdot \mathrm{min}^{-1}$, the nondominant forearm was exercised at power levels of $6,12,24$, and $36 \mathrm{~J} \cdot \mathrm{min}^{-1}$. The duration of each workload after warm-up was $10 \mathrm{~min}$ to allow for $5 \mathrm{~min}$ of equilibration and 5 min of steady-state data collection. The same protocol was repeated with the dominant forearm. After the 28-d training program, each subject was retested using the identical protocol.

Forearm blood flow. Venous occlusion plethysmography was used to measure skeletal muscle blood flow (12-16). Typically, this technique uses two inflation cuffs, with the upper cuff secured as far from the olecranon process as possible and the lower cuff secured around the wrist. To ensure adequate venous drainage, the arm rests on a board and is positioned $10 \mathrm{~cm}$ above the level of the right atrium. When the upper cuff is inflated to $40 \mathrm{mmHg}$ (venous occlusive pressure), venous outflow is obstructed, and the volume of the forearm increases due to 
arterial inflow. Since the shape of the forearm approximates a cylinder, the change in circumference measured by a mercury-in-silastic strain gauge positioned around the forearm is directly proportional to a change in volume and, therefore, blood flow.

For resting blood flow measurements, the wrist cuff was inflated to $240 \mathrm{mmHg}$ for $1 \mathrm{~min}$ before flow measurements to eliminate venous return from the hand. Arterial inflow tracings were then obtained by inflating the upper cuff to $\mathbf{4 0} \mathrm{mmHg}$ to occlude the venous system. Three resting blood flow determinations were averaged. Resting flow was calculated as milliliters per $100 \mathrm{ml}$ of forearm volume per min $\left(\mathrm{ml} \cdot 100 \mathrm{ml}^{-1} \cdot \mathrm{min}^{-1}\right)$.

Exercise forearm blood flow was measured without the wrist cuff (13-15). The strain gauge was secured on the forearm $1 \mathrm{~cm}$ proximal to the ${ }^{31}$ P-MRS sample volume. A rapid inflation cuff was then secured around the upper arm, and the arm was positioned in the bore of the magnet at approximately the same height as the shoulder to facilitate venous return (Fig. 1). The cuff was inflated to $40 \mathrm{mmHg}$ and arterial inflow tracings were obtained within $2 \mathrm{~s}$ after the final exercise contraction at workloads of 12,24 , and $36 \mathrm{~J} \cdot \mathrm{min}^{-1}$. Exercise flow was calculated and expressed in the same manner as resting flows.

Forearm muscle mass. Relative muscle mass in the forearm before and after training was determined by magnetic resonance imaging with a 1.5-T imaging system (General Electric, Milwaukee, WI) $(14,15)$. Two-dimensional transverse images were obtained from 3-mm-thick slices in the same location on each forearm, approximately one-third the ulnar length distal to the olecranon process (Fig. 2). A spin echo sequence with pulse repetition time of $300 \mathrm{~ms}$, echo time of $20 \mathrm{~ms}$, acquisition matrix of $256 \times 256$, and a 10-cm field of view was used without signal averaging. Images were analyzed with area tracing software. Cross-sectional area of the flexor muscle group was measured before and after training. Bone and subcutaneous fat were excluded from area measurements, but vascular and nerve areas were small and not separated from surrounding tissue. It was assumed that the crosssectional area was proportional to muscle mass $(14,15)$

Maximal voluntary contraction. Maximal voluntary contraction strength of the wrist flexors was tested on an arm exercise apparatus identical to the one used in the magnet. With the arm positioned as it was for the magnet studies, subjects performed a maximal isometric wrist flexion contraction with the handle at the midpoint of the range of motion. Three maximal efforts were performed, separated by at least $15 \mathrm{~s}$ of rest. The highest force of these three contractions was defined as maximal voluntary contraction.

Endurance testing. Forearm endurance was determined in both forearms before and after the 28-d training program. Subjects performed the endurance test two times before training was begun, the first test serving only to familiarize the subjects with the test routine. 2 $d$ of rest intervened between these two endurance tests. The endurance test protocol was as follows: with the arm in the same position as that
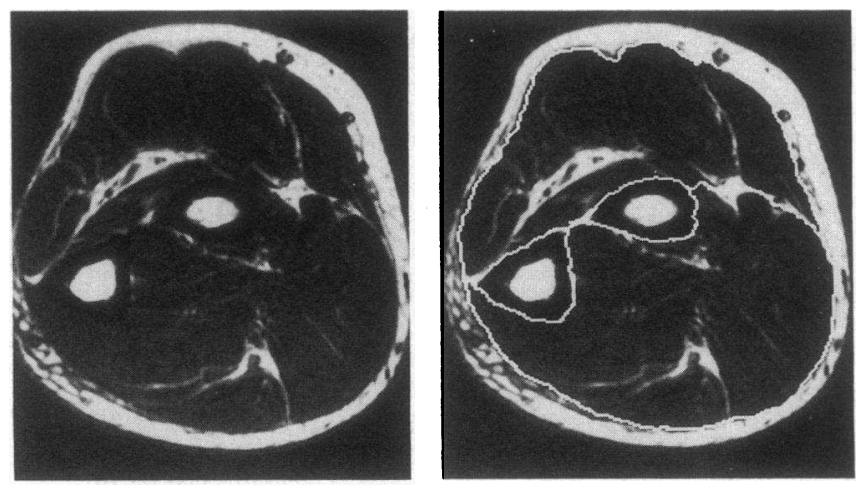

Figure 2. Forearm cross-sectional images were obtained by magnetic resonance imaging before and after training. Flexor muscle area was measured by planimetry of the flexor muscle group. Bone and subcutaneous fat were excluded from the area measurements. used for training (see section below), subjects performed wrist flexion (two repetitions every $5 \mathrm{~s}$ ) with a 1.9-kg weight until exhaustion. The endurance protocol was performed first by the nondominant forearm, then by the dominant forearm, with a 15-min rest period intervening. Exhaustion was defined as the inability to lift the weight through a full range of motion for two successive repetitions. Maximal endurance of the forearm was recorded as the time to exhaustion.

Systemic exercise testing. Upright exercise testing was performed using an electronically braked cycle ergometer (Erich Jaeger, Rockford, IL) that maintains constant workload at pedal frequencies of 40-100 rpm. Testing was performed in an air-conditioned laboratory with an ambient temperature of $22-24^{\circ} \mathrm{C}$ and humidity of $30-40 \%$. Before exercise, subjects rested upright on the cycle ergometer for 3-5 min. Exercise was initially performed unloaded and then increased by $40 \mathrm{~W}$ every $3 \mathrm{~min}$. Exercise was continued until exhaustion, which was defined as the inability to maintain critical pedal frequency $(>40$ rpm). Standard verbal encouragement was used for all subjects. Respiratory gas exchange was measured continuously (Ergopneumotest, Erich Jaeger). Peak $\mathrm{VO}_{2}$ was defined as the highest $\mathrm{VO}_{2}$ reached during the exercise test.

Training program. Each training session was supervised by either a physician or an exercise physiologist involved in the study. The 28-d training program consisted of wrist flexion exercise performed with the nondominant forearm six times a week using a handheld weight. The weights were cylindrical plastic containers measuring $5.5 \times 11 \mathrm{~cm}$ filled with lead shot to attain the desired weight and padding to stabilize the shot. The workloads were the same $(1.9 \mathrm{~kg})$ for each subject and were not changed during training.

Daily training consisted of three 8-min exercise sets separated by 5-min rest periods. Exercise was performed in a sitting position with the dorsal aspect of the forearm stabilized on a flat surface well below the level of the shoulder. The dorsal aspect of the wrist was supported by a pad to allow hyperextension. The weight was lifted through the entire range of motion and returned to the original position once every $5 \mathrm{~s}$. Subjects were instructed to place the nonexercising arm in a comfortable position and to avoid any muscular contraction in that arm. If any contraction of the non-exercising forearm could be palpated by the observer, the patient was instructed to relax that arm.

During the first week of training, exercise cardiac output, heart rate, plasma lactate, and norepinephrine were determined during a training session to assess the systemic impact of the localized training.

Cardiac output and heart rate. Heart rate and blood flow velocity in the ascending aorta were measured by $3 \mathrm{mHz}$ continuous wave Doppler ultrasound velocimetry (Quinton ExerDop; Quinton Instrument Co., Seattle, WA) at rest and during minute 6 of each of the three training sets. The Doppler transducer was hand held in the suprasternal notch, with the ultrasound beam directed inferiorly and anteriorly into the flow stream of the ascending aorta and was manipulated to obtain the strongest audio signal. The Doppler-shifted velocity signals were integrated by a microprocessor within the ExerDop instrument to obtain values for beat-to-beat stroke distance. Cardiac output was estimated from the product of stroke distance, heart rate, and an aortic cross-sectional area that was determined by standard two-dimensional echocardiography on each patient. The aortic cross-sectional area was measured just distal to the aortic bulb.

Lactate measurements. Measurements of lactate were made at rest in duplicate and during minute 7 of the first and third exercise sets of one training session. Approximately $3 \mathrm{ml}$ of blood were collected in vacuum tubes containing potassium oxalate and sodium fluoride. Samples were placed on ice until analysis, which was performed $\sim 10$ min after exercise ended. $25 \mu$ l blood were extracted with a calibrated syringe pipette and injected into an automated lactate analyzer (model 23L; Yellow Springs Instrument Co., Yellow Springs, $\mathrm{OH}$ ) for determination of extracellular lactate concentration. The drift was $<0.1 \mathrm{~mm} /$ liter after any measurement series.

Plasma norepinephrine. Immediately after collection of blood for lactate analysis, a 4-ml venous blood sample was drawn and placed in a chilled heparinized collection tube containing $6 \mathrm{mg}$ of reduced gluta- 


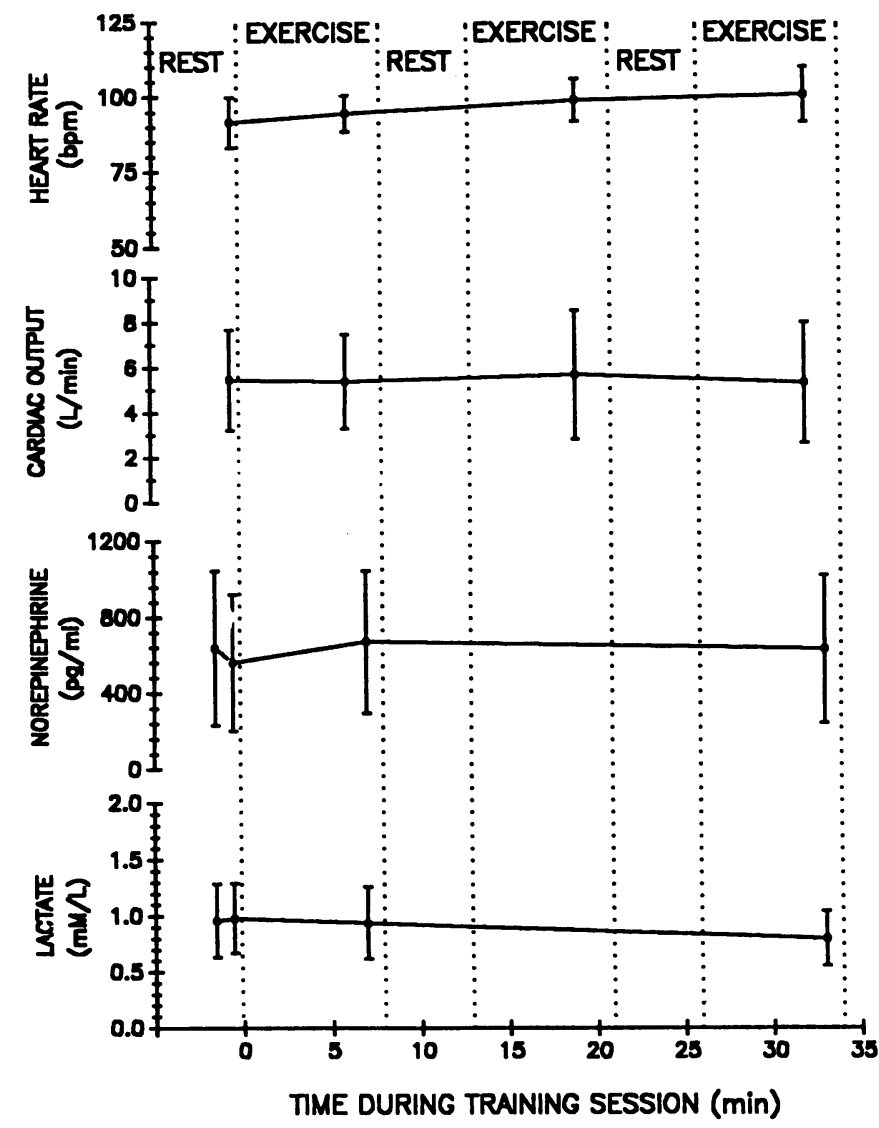

Figure 3. Heart rate and cardiac output were measured once at rest and three times during exercise. Plasma norepinephrine and lactate were measured in duplicate at rest and twice during exercise in the nonexercising arm. No significant change in any of these parameters was detectable by one-way analysis of variance for repeated measures.

thione. Contents of the tube were mixed gently and then placed on ice. Within $30 \mathrm{~s}$ of collection, the samples were centrifuged at $3,000 \mathrm{rpm}$ for $15 \mathrm{~min}$ at $4^{\circ} \mathrm{C}$. The plasma was placed in polypropylene tubes and stored at $-70^{\circ} \mathrm{C}$. Samples were then analyzed for norepinephrine using a high performance liquid chromatograph (Beckman Instruments, Fullerton, CA) and a plasma catecholamine analysis kit (ESA, Inc., Bedford, MA).

Statistical methods. Data were entered into a microcomputer data base and analyzed by a commercial statistical package (Systat, Inc., Evanston, IL). The effect of training on metabolic and hemodynamic parameters was analyzed by paired $t$ test. Linear regression was used to examine the relationship between the $\mathrm{P}_{\mathrm{i}} / \mathrm{PCr}$ ratio and workload. Changes in hemodynamic and metabolic parameters during the training protocol were examined by one-way analysis of variance for repeated measures. Statistical significance was assumed for $P$ values $\leq 0.05$ (17).

\section{Results}

Hemodynamic and hormonal changes during training sessions. To determine whether the localized training regimen produced systemic hemodynamic, hormonal, or metabolic effects, heart rate, cardiac output, plasma norepinephrine, and lactate were measured before and during a training session (Fig. 3). None of the measured variables were elevated significantly from rest during this session.

Forearm metabolic changes during exercise and effects of training. Fig. 4 shows a series of typical spectra obtained at rest and during three progressive exercise workloads. The $\mathrm{P}_{\mathrm{i}}, \mathrm{PCr}$, as well as the three ATP peaks $(\alpha, \beta, \gamma)$ are clearly visible. This example demonstrates the increase in $\mathrm{P}_{\mathrm{i}}$ and decrease in $\mathrm{PCr}$ that occurs with progressive exercise. However the relationship of $\mathrm{P}_{\mathrm{i}} / \mathrm{PCr}$ versus workload was not linear in all patients.

Forearm blood flow and ${ }^{31} \mathrm{P}-\mathrm{MRS}$ data were collected from each forearm at rest and during exercise at submaximal workloads. The exercise protocols in the magnet were performed without incident. One subject (S.B.) was unable to complete the $36 \mathrm{~J} \cdot \mathrm{min}^{-1}$ workload in either forearm due to muscle fatigue. Subject R.R. failed to reach a steady-state $\mathrm{P}_{\mathrm{i}} / \mathrm{PCr}$ at 36 $\mathrm{J} \cdot \mathrm{min}^{-1}$ in the nondominant forearm. Data from these workloads were not included in the analyses. The $\mathrm{P}_{\mathrm{i}} / \mathrm{PCr}$ ratios calculated from the nondominant and dominant forearm data increased in each individual with progressive exercise workloads before and after training (Table III, Fig. 5). The mean slope of $\mathrm{P}_{\mathrm{i}} / \mathrm{PCr}$ vs workload before training in the nondominant forearm in our patients is similar (after normalizing units of workload) to that previously reported by other investigators in patients with heart failure (7). In each subject, the $P_{i} / P C r$ slope decreased significantly in the nondominant forearm after training. The $\mathrm{P}_{\mathrm{i}} / \mathrm{PCr}$ slope in the untrained forearm was not significantly changed by nondominant forearm training. Since the $\mathrm{P}_{\mathrm{i}} / \mathrm{PCr}$ ratio is affected by the hydrogen ion concentration, these data were also examined after adjusting for changes in pH. The significance of the decreased relationship of $\mathrm{P}_{\mathrm{i}} / \mathrm{PCr}$ versus workload relationship in the nondominant forearm after training was unchanged by this calculation $(P=0.015)$.

Intracellular $\mathrm{pH}$, calculated from the chemical shift difference between $P_{i}$ and $\mathrm{PCr}$ (Eq. 1), declined at the higher levels of exercise, but was not affected by training (Table IV). The concentration of ATP at each exercise workload did not vary significantly in either forearm from the assumed resting value of $8.2 \mathrm{mM}$ (11). The mean exercise ATP value in the nondominant forearm was $8.9 \pm 2.2$ before and $8.8 \pm 1.4 \mathrm{mM}$ after training; in the dominant forearm the mean value $8.7 \pm 0.9$ before and $8.6 \pm 1.5 \mathrm{mM}$ after training.
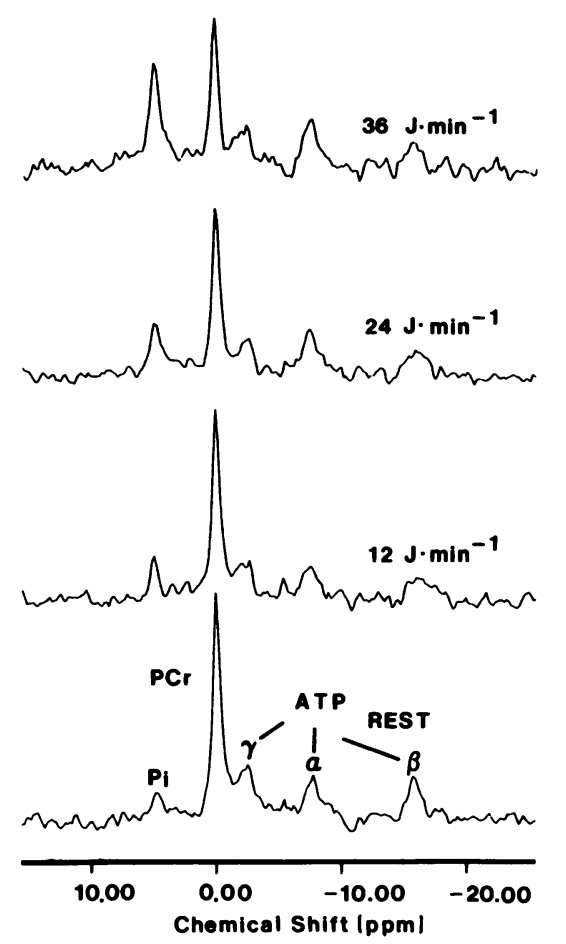

Figure 4. Characteristic ${ }^{31}$ P-MRS spectra obtained during rest and progressive workloads. Peaks corresponding to $P_{i}, P C r$, and ATP were resolved. Progressive exercise resulted in depletion of PCr and increased $P_{i}$. The chemical shift, in parts per million, between $\mathrm{P}_{\mathrm{i}}$ and $\mathrm{PCr}$ was used to calculate intracellular pH. 


\begin{tabular}{lcccc}
\hline & \multicolumn{2}{c}{ Nondominant $P_{i} / P C r$} & & Dominant $P_{i} / P C r$ \\
\cline { 2 - 3 } \multicolumn{1}{c}{ Workload } & Before & After & Before & \\
\hline \multicolumn{1}{c}{$J \cdot$ min $^{-1}$} & & & & \\
Rest & $0.19 \pm 0.03$ & $0.20 \pm 0.03$ & $0.19 \pm 0.03$ & $0.18 \pm 0.02$ \\
6 & $0.27 \pm 0.07$ & $0.25 \pm 0.05$ & $0.22 \pm 0.07$ & $0.22 \pm 0.06$ \\
12 & $0.33 \pm 0.05$ & $0.30 \pm 0.12$ & $0.31 \pm 0.09$ & $0.27 \pm 0.05$ \\
24 & $0.97 \pm 0.39$ & $0.62 \pm 0.16$ & $0.54 \pm 0.11$ & $0.47 \pm 0.06$ \\
36 & $1.65 \pm 0.25^{*}$ & $0.99 \pm 0.17^{*}$ & $1.23 \pm 0.56^{\ddagger}$ & $0.84 \pm 0.12^{\ddagger}$ \\
Regression slope & $0.050 \pm 0.010^{\S}$ & $0.028 \pm 0.006^{\S}$ & $0.030 \pm 0.017^{\prime \prime}$ & $0.020 \pm 0.006^{\prime \prime}$ \\
$r$ & $0.93 \pm 0.04$ & $0.95 \pm 0.03$ & $0.94 \pm 0.02$ & $0.97 \pm 0.03$ \\
\hline
\end{tabular}

Values reported as mean \pm SD. $n=5$ except where indicated; ${ }^{*} n=3 ;{ }^{\ddagger} n=4 .{ }^{8}$ Statistically significant difference $(p<0.02)$ after training; ${ }^{\prime \prime}$ difference not significant.

Changes in exercise endurance. Every subject demonstrated an increase in nondominant forearm endurance after training. This increase in the mean endurance was $>260 \%$ and was statistically significant (Fig. 6). In contrast, there was no significant change in endurance in the untrained forearm.

Forearm blood flow and indicators of muscle mass. Forearm blood flow increased with progressive exercise, but was the same before and after training in each forearm (Table IV). Training also had no effect on flexor muscle cross-sectional area as measured by magnetic resonance imaging, or on maximal voluntary contraction (Table V).

Oxygen uptake. Peak systemic oxygen consumption demonstrated a small (mean peak $\mathrm{VO}_{2}$ before $15.5 \pm 4.5 \mathrm{vs}$. after
$16.4 \pm 4.2 \mathrm{ml} \cdot \mathrm{kg} \cdot \mathrm{min}^{-1}$; mean difference $0.88 \pm 0.36$ ) increase after training. Although statistically significant, the physiological relevance of such a small change is unclear.

\section{Discussion}

Exercise intolerance is the major complaint of patients with mild and moderate CHF (1-3). Despite high ventricular filling pressures and frequent complaints of dyspnea on exertion, the most common limiting symptom during maximal exercise testing is the subjective complaint of muscle fatigue (3). Intuitively, one would expect the severity of exercise impairment to parallel the degree of left ventricular dysfunction; however,
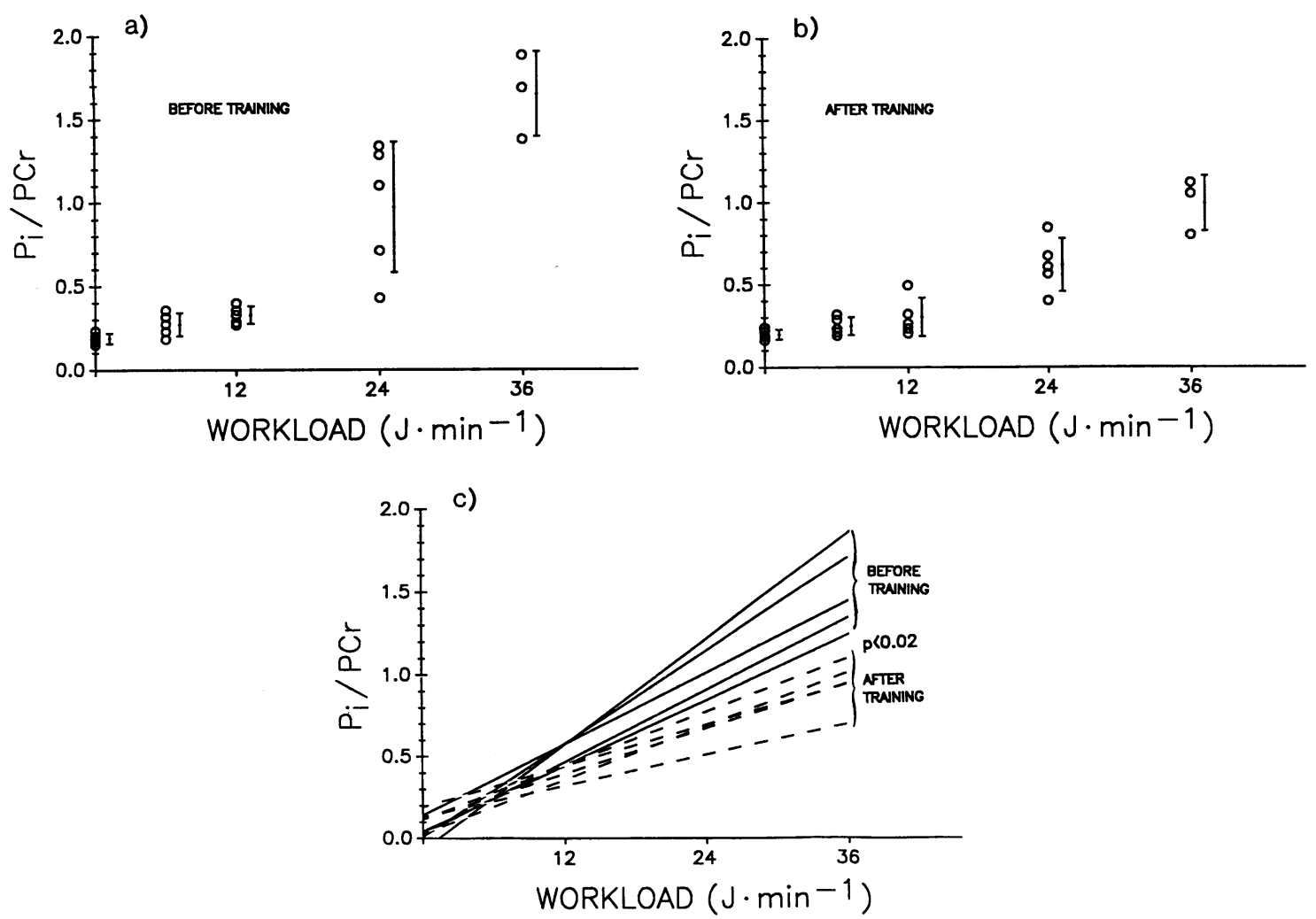

Figure 5. (a) This graph represents the individual values, mean, and the SD of the $\mathrm{P}_{\mathrm{i}} / \mathrm{PCr}$ ratio versus workload of the nondominant forearm of each subject before training, and $(b)$ after training. $(c)$ The regression lines of the relationship of $\mathrm{P}_{\mathrm{i}} / \mathrm{PCr}$ vs submaximal workloads are plotted for each patient before and after training. Training resulted in a decreased slope in each patient $(n=5$, paired $t$ test). 
Table IV. Effect of Training on pH and Forearm Blood Flow

\begin{tabular}{|c|c|c|c|c|}
\hline \multicolumn{3}{|c|}{ Nondominant forearm } & \multicolumn{2}{|c|}{ Forearm blood flows } \\
\hline Workload & Before & After & Before & After \\
\hline \multicolumn{5}{|l|}{$J \cdot \min ^{-1}$} \\
\hline Rest & $7.05 \pm 0.08$ & $7.02 \pm 0.06$ & $3.0 \pm 0.8$ & $3.3 \pm 0.8$ \\
\hline 6 & $7.03 \pm 0.12$ & $7.01 \pm 0.06$ & - & - \\
\hline 12 & $7.03 \pm 0.11$ & $6.97 \pm 0.04$ & $6.9 \pm 1.9$ & $6.5 \pm 1.4$ \\
\hline 24 & $6.90 \pm 0.07$ & $6.94 \pm 0.05$ & $7.4 \pm 1.4$ & $8.5 \pm 4.8$ \\
\hline $36^{*}$ & $6.85 \pm 0.10$ & $6.85 \pm 0.11$ & $15.4 \pm 4.3$ & $17.0 \pm 7.3$ \\
\hline \multicolumn{5}{|c|}{ Dominant forearm } \\
\hline Rest & $7.04 \pm 0.06$ & $7.02 \pm 0.06$ & $4.4 \pm 3.3$ & $2.8 \pm 0.6$ \\
\hline 6 & $7.04 \pm 0.07$ & $7.04 \pm 0.08$ & - & - \\
\hline 12 & $6.98 \pm 0.03$ & $7.03 \pm 0.08$ & $8.9 \pm 4.1$ & $7.3 \pm 3.1$ \\
\hline 24 & $6.99 \pm 0.08$ & $6.96 \pm 0.06$ & $8.2 \pm 3.5$ & $8.6 \pm 4.0$ \\
\hline $36^{\ddagger}$ & $6.93 \pm 0.08$ & $6.91 \pm 0.12$ & $12.1 \pm 4.4$ & $12.8 \pm 6.2$ \\
\hline
\end{tabular}

Mean \pm SD. $n=5$ except where indicated. ${ }^{*} n=3 ;{ }^{\ddagger} n=4 .{ }^{\S}$ Units of measure used are milliliters $\cdot 100$ milliliters tissue volume $\cdot \mathrm{min}^{-1}$.

a consistent finding in CHF is that exercise capacity and indices of left ventricular performance correlate poorly (2). These observations raise the possibility that peripheral changes are the major determinant of exercise performance in chronic CHF.

The peripheral abnormality initially demonstrated in $\mathrm{CHF}$ was a reduction in blood flow to exercising limbs during both submaximal and maximal exertion (18). Subsequently, abnormalities in skeletal muscle morphology and metabolism have been described by a number of investigators. Although a consistent pattern of change has not been identified, biopsy studies have reported decreased oxidative enzyme activity and muscle fiber atrophy $(5,19,20)$. Abnormalities in skeletal muscle bioenergetics have also been described. Using ${ }^{31} \mathrm{P}-\mathrm{MRS}$, Wilson et al. (7) demonstrated excessive depletion of $\mathrm{PCr}$ and greater acidosis in the forearm muscles during submaximal exercise in CHF patients when compared with normal subjects. These findings were later confirmed by Massie et al. $(8,9)$, who also found a relationship between the degree of skeletal muscle

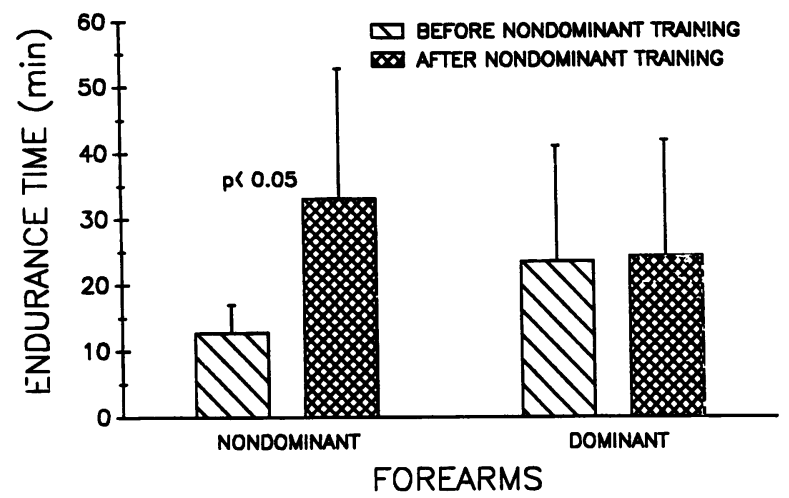

Figure 6. Endurance times in the nondominant and dominant forearms were tested before and after training at the same absolute submaximal workload. Endurance in the trained nondominant forearm was increased ( $n=5$, paired $t$ test). Endurance in the dominant forearm was not increased.
Table V. Effects of Training on Maximal Voluntary Contraction and Flexor Muscle Cross-Sectional Area

\begin{tabular}{|c|c|c|c|c|}
\hline \multicolumn{3}{|c|}{ Nondominant forearm } & \multicolumn{2}{|c|}{$\begin{array}{c}\text { Flexor muscle } \\
\text { cross-sectional area }\end{array}$} \\
\hline \multirow[t]{2}{*}{ Subject } & Before & After & Before & After \\
\hline & & & \multicolumn{2}{|c|}{$\mathrm{cm}^{2}$} \\
\hline P.V. & 87 & 100 & 18.2 & 18.4 \\
\hline R.R. & 108 & 80 & 18.9 & 19.5 \\
\hline E.T. & 155 & 174 & 20.4 & 20.3 \\
\hline J.K. & 228 & 255 & 19.4 & 20.3 \\
\hline S.B. & 174 & 160 & 19.8 & 19.7 \\
\hline Mean \pm SD & $150 \pm 56$ & $154 \pm 69$ & $19.3 \pm 0.8$ & $19.6 \pm 0.8$ \\
\hline \multicolumn{5}{|c|}{ Dominant forearm } \\
\hline P.V. & 97 & 106 & 19.3 & 18.7 \\
\hline R.R. & 123 & 104 & 20.0 & 20.1 \\
\hline E.T. & 140 & 155 & 20.3 & 20.3 \\
\hline J.K. & 263 & 222 & 24.3 & 24.8 \\
\hline S.B. & 154 & 184 & 19.0 & 18.7 \\
\hline Mean \pm SD & $155 \pm 64$ & $154 \pm 51$ & $20.4 \pm 1.7$ & $20.5 \pm 2.5$ \\
\hline
\end{tabular}

MVC, maximal voluntary contraction.

abnormalities in the forearm during exercise and the clinical severity of $\mathrm{CHF}$. Furthermore, these changes appeared to be unrelated to limb blood flow and were present during ischemic as well as aerobic exercise (9).

Despite the prevalence of skeletal muscle abnormalities, there is no evidence to indicate that they are primary events in CHF. Most likely, the initial change is a reduction of blood flow to exercising skeletal muscle due to reduced cardiac output reserve and perfusion pressure. In addition, there are alterations in sympathetic nervous system outflow to muscle and reduced responsiveness to catecholamines (21). We suspect that these changes decrease aerobic metabolism and work capacity, resulting in reduced physical activity, thereby initiating a cycle of deconditioning and further inactivity.

Since deconditioning may be responsible for a large part of the skeletal muscle abnormalities seen in CHF, defining the metabolic and flow responses to exercise training may further elucidate the pathophysiology of these abnormalities and reveal possible therapeutic interventions. A recent study (10) has examined the hemodynamic, metabolic, and clinical response to systemic exercise training in patients with CHF. After 4-6 mo of systemic training, 9 out of 12 patients with stable CHF exhibited a significant increase in peak $\mathrm{VO}_{2}$, even though central hemodynamics did not improve. However, there were a number of significant changes in the exercising limb, including an increase in blood flow, oxygen uptake, and arteriovenous oxygen difference at maximal exercise, and a decrease in lactate accumulation during submaximal exercise. These peripheral changes suggest an improved skeletal muscle metabolic response to exercise. Since systemic training was used, it is difficult to attribute these changes solely to peripheral adaptations. The purpose of this study was to isolate the peripheral effect of exercise training. The exercise performed in this study was designed to focus on the forearm flexor muscles without eliciting a systemic cardiovascular response. Furthermore, by 
evaluating both the trained and nontrained limb, we controlled for motivational factors.

Our results demonstrate that a localized training regimen can improve the metabolic and functional capacity of skeletal muscle in patients with CHF. After $28 \mathrm{~d}$ of forearm training, the trained forearm demonstrated greater submaximal endurance and a lower $\mathrm{P}_{\mathrm{i}} / \mathrm{PCr}$ ratio as a function of submaximal workload. This suggests an improved peripheral response to exercise with training.

The low workloads used in the training program did not elicit a systemic cardiovascular response, as reflected by the lack of significant increases in heart rate, cardiac output, plasma lactate, and norepinephrine during training. This is further confirmed by the fact that the untrained limb did not exhibit a change in either exercise capacity or muscle metabolism after training. The small increase in peak systemic $\mathrm{VO}_{2}$ at the end of the protocol is the one discordant finding. This may reflect an improvement in overall cardiac status as a result of one month of careful medical management, but more likely reflects the usual modest increase in peak $\mathrm{VO}_{2}$ that is observed in serial testing of patients with CHF.

Several mechanisms that could explain the training-induced improvement in muscle energetics during exercise warrant consideration. These include an increase in muscle mass, improvements in oxygen delivery, altered neural regulation leading to changes in motor unit recruitment, and training-induced biochemical adaptations resulting in greater oxidative capacity in the exercising muscle.

An increase in muscle mass would allow the same amount of work to be performed at a lower intensity per volume of muscle. Similarly, if strength improved with training, the same absolute workload would represent a lower percentage of maximum. The decrease in relative work intensity that would result from changes in either muscle mass or strength would predictably improve metabolism during constant submaximal exercise loads. However, muscle mass, estimated by MRI, and strength, measured as maximal voluntary contraction, were both unaffected by training. Therefore, changes in either muscle mass or strength are unlikely to account for the observed improvement in muscle $\mathrm{P}_{\mathrm{i}} / \mathrm{PCr}$ during exercise.

Increased blood flow after training also could have affected the metabolic response to exercise. During submaximal exercise, a reduction of oxygen delivery to exercising skeletal muscle results in a higher $\mathrm{P}_{\mathrm{i}} / \mathrm{PCr}$ and greater lactate production (22). Since the peripheral blood flow response to exercise in CHF is inappropriately low $(18,23)$, an increase in blood flow could be expected to improve exercise metabolism. However, we found that forearm blood flow during submaximal exercise was not changed by training in these patients. This is consistent with studies in normal subjects $(15,24-26)$ and in CHF patients (10), which have shown that peripheral blood flow at a given submaximal workload is unchanged or decreased after training. Although we cannot exclude a redistribution of flow within the forearm to more effectively perfuse the exercising muscles (27), the important point for patients with compromised central circulatory function is that improvements in exercising muscle metabolism can be achieved without requiring increased limb blood flow and, therefore, increased cardiac output.

Neural adjustments also could have contributed to the decrease in exercise $P_{i} / P C r$ after training. Alterations in recruitment of motoneurons as a result of endurance training have not been well defined, but some adaptations are likely (28).
Certainly, some forms of training result in neural changes. Gains in strength induced by weight training are primarily due to alterations in neural recruitment in the first weeks of training and to muscle hypertrophy after that time (29). Since motor units are recruited in an orderly fashion depending on the orientation and force of movement (28), the possibility of altered recruitment patterns was minimized by exercising each forearm in an identical manner at the same absolute workloads before and after training. Also, strength was not increased by our training program. Therefore, we suspect only a minor neural contribution, if any, to the lower exercise $\mathrm{P}_{\mathrm{i}} / \mathrm{PCr}$ ratio observed.

It is likely that an increase in the oxidative capacity of muscle is responsible for the lower $P_{i} / P C r$ versus workload relationship in our patients after training. Terjung et al. (30) demonstrated that the concentration of free ADP during steady-state muscle contraction is altered by the mitochondrial content of skeletal muscle. Inducing hypothyroidism in rats decreased the mitochondrial content and raised the free ADP levels at a given oxygen consumption compared with controls. In contrast, training increased mitochondrial content and lowered free ADP concentration at the same oxygen consumption.

Direct noninvasive measurement of ADP is not possible with existing technology. However, through the creatine kinase equilibrium reaction, ADP can be calculated from cellular creatine, PCr, hydrogen ion, and ATP levels. Fortunately, during submaximal steady-state exercise, ATP and hydrogen ions remain nearly constant, while creatine can be approximated with cellular $P_{i}$, making the $P_{i} / P C r$ ratio determined by ${ }^{31} \mathrm{P}-\mathrm{MRS}$ an indicator of free $\operatorname{ADP}(31,32)$.

Our subjects were tested at the same workload and, thus, at the same oxygen consumption before and after training. Therefore, the lower exercise $\mathrm{P}_{\mathrm{i}} / \mathrm{PCr}$ seen in our subjects after training is consistent with an increased oxidative capacity in the forearm skeletal muscle.

The finding of most potential clinical importance in this study is the marked improvement in forearm endurance seen after training. The mechanism responsible for the extended endurance most likely was an improvement in oxidative capacity of the trained skeletal muscle (33). Although systemic training improves the oxidative capacity of skeletal muscle, there are potential disadvantages of systemic training in $\mathrm{CHF}$ patients (10). The enlarged left ventricular end-diastolic volume characteristic of patients with $\mathrm{CHF}$ may worsen during systemic exercise. This can contribute to increased wall stress and further compromise of systolic function. Furthermore, some patients are unable to perform regular systemic exercise at an intensity sufficient to provide a training stimulus. Thus, systemic training in this subset of patients may not only have limited effectiveness, but may be potentially harmful.

We conclude that skeletal muscle in CHF patients can adapt metabolically and functionally to localized training. The lower $\mathrm{P}_{\mathrm{i}} / \mathrm{PCr}$ as a function of submaximal workload in the trained muscle occurred without associated changes in muscle mass, forearm blood flow, or the systemic response during exercise training. The lower $\mathrm{P}_{\mathrm{i}} / \mathrm{PCr}$ is associated with improved forearm endurance and likely reflects an improved oxidative capacity of muscle. We postulate that by applying localized peripheral training to larger muscle groups, such as the leg, and training each leg in sequential fashion, submaximal systemic exercise performance in patients with CHF may be improved without the disadvantages of systemic exercise. 


\section{Acknowledgments}

The authors wish to thank Julia Martin and Henrietta Aguilar for secretarial support and Frank Gurule for technical assistance.

This work was supported in part by the New Mexico affiliate of the American Heart Association, a grant from the General Clinical Research Program, National Institutes of Health grant DRR 5 MOI RR00997-14, and the Veterans Administration.

\section{References}

1. Franciosa, J. A., S. Ziesche, and M. Wilen. 1979. Functional capacity of patients with chronic left ventricular failure: relationship of bicycle exercise performance to clinical and hemodynamic characterization. Am. J. Med. 67:460-466.

2. Szlachcic, J., B. M. Massie, B. L. Kramer, N. Topic, and J. Tubau. 1985. Correlates and prognostic implication of exercise capacity in chronic congestive heart failure. Am. J. Cardiol. 55:1037-1042.

3. Wilson, J. R., and N. Ferraro. 1983. Exercise intolerance in patients with congestive heart failure: relationship to oxygen transport and ventilatory abnormalities. Am. J. Cardiol. 51:1358-1363.

4. Wilson, J. R., J. L. Martin, and N. Ferraro. 1984. Impaired skeletal muscle nutritive flow during exercise in patients with congestive heart failure: Role of cardiac pump dysfunction as determined by the effect of dobutamine. Am. J. Cardiol. 53:1308.

5. Lipkin, D. P., D. A. Jones, J. M. Round, and P. A. Poole-Wilson. 1988. Abnormalities of skeletal muscle in patients with chronic heart failure. Int. J. Cardiol. 18:187-195.

6. Weiner, D. H., L. I. Fink, J. Maris, R. A. Jones, B. Chance and J. R. Wilson. 1986. Abnormal skeletal muscle bioenergetics during exercise in patients with heart failure: role of reduced muscle flow. Circulation. 73:1127-1136.

7. Wilson, J. R., L. Fink, J. Maris, N. Ferraro, J. Power-Vanwart, S. Eleff, and B. Chance. 1985. Evaluation of energy metabolism in skeletal muscle of patients with heart failure with gated phosphorus-31 nuclear magnetic resonance. Circulation. 71:57-62.

8. Massie, B. M., M. Conway, R. Yonge, S. Frostick, J. Ledingham, P. Sleight, G. Radda, and B. Rajagopalan. 1987. Skeletal muscle metabolism in patients with congestive heart failure. Relation to clinical severity and blood flow. Circulation. 76:1009-1014.

9. Massie, B. M., J. Conway, B. Rajagopalan, R. Yonge, S. Frostick, J. Ledingham, P. Sleight, and G. Radda. 1988. Skeletal muscle metabolism during exercise under ischemic conditions in congestive heart failure. Evidence for abnormalities unrelated to blood flow. Circulation. 78:320-326.

10. Sullivan, M. J., M. B. Higginbotham, and F. R. Cobb. 1988. Exercise training in patients with severe left ventricular dysfunction. Hemodynamic and metabolic effects. Circulation. 78:506-515.

11. Taylor, D. J., P. Styles, P. M. Mathews, D. A. Arnold, D. G. Gadian, P. Bore, and G. K. Radda. 1986. Energetics of human muscle: exercise-induced ATP depletion. Magn. Reson. Med. 3:44-54.

12. Burger, H. C., H. W. Horeman, and A. J. M. Brakkee. 1959. Comparison of some methods for measuring peripheral blood flow. Phys. Med. Biol. 4:168-175.

13. Whitney, R. J. 1953. Measurement of volume changes in human limbs. J. Physiol. (Lond.). 121:1-27.

14. Minotti, J. R., E. C. Johnson, T. L. Hudson, R. R. Sibbitt, L. E. Wise, E. Fukushima, and M. V. Icenogle. 1989. Forearm metabolic asymmetry detected by ${ }^{31} \mathrm{P}$ NMR during submaximal exercise. J. Appl. Physiol. 67:324-329.

15. Minotti, J. R., E. C. Johnson, T. L. Hudson, G. Zuroske, E. Fukushima, G. Murata, L. E. Wise, T. W. Chick, and M. V. Icenogle.
1990. Training-induced skeletal muscle adaptations are independent of systemic adaptations. J. Appl. Physiol. 68:289-294.

16. Sinoway, L., J. Minotti, D. Davis, J. Pennock, J. Burg, T. Musch, and R. Zelis. 1988. Delayed reversal of impaired vasodilation in congestive heart failure after heart transplantation. Am. J. Cardiol. 61:1076-1079.

17. Zar, J. H. 1974. Biostatistical Analysis. Prentice-Hall, Inc., Englewood Cliffs, NJ. 43-45.

18. Zelis, R., J. Longhurst, R. J. Capone, and D. T. Mason. 1974. A comparison of regional blood flow and oxygen utilization during dynamic forearm exercise in normal subjects and patients with congestive heart failure. Circulation. 50:137-143.

19. Sullivan, M. J., Higginbotham, H. J. Green, and F. R. Cobb. 1989. Decreased aerobic oxidative capacity in chronic heart failure. JACC (J. Am. Coll. Cardiol.). 13:39A. (Abstr.)

20. Yancy, C. W. Jr., D. Parsons, L. Lane, M. Carry, B. G. Firth, and G. Blomqvist. 1989. Capillary density, fiber type, and enzyme composition of skeletal muscle in congestive heart failure. JACC ( $J$. Am. Coll. Cardiol.). 13:38A. (Abstr.)

21. Frey, M. J., V. Lance, N. Ferraro, P. B. Molinoff, and J. R. Wilson. 1988. Down-regulation of skeletal muscle beta-adrenergic receptors in experimental canine heart failure. JACC (J. Am. Coll. Cardiol.). 11:116A. (Abstr.)

22. Weiner, D. H., J. Maris, B. Chance, and J. R. Wilson. 1986. Detection of skeletal muscle hypoperfusion during exercise using phosphorus-31 nuclear magnetic resonance spectroscopy. JACC (J. Am. Coll. Cardiol.). 7:793-799.

23. Zelis, R., D. T. Mason, and E. Braunwald. 1968. A comparison of peripheral resistance vessels in normal subjects and in patients with congestive heart failure. J. Clin. Invest. 47:960-969.

24. Bergman, H., P. Bjorntorp, T.-B. Conradson, M. Fahlen, J. Stenberg, and E. Varnauskas. 1973. Enzymatic and circulatory adjustments to physical training in middle-aged men. Eur. J. Clin. Invest. 3:414-418.

25. Saltin, B., K. Nazar, D. L. Costill, E. Stein, E. Jansson, B. Essen, and P. D. Gollnick. 1976. The nature of the training response; peripheral and central adaptations to one-legged exercise. Acta Physiol. Scand. 96:289-305.

26. Varnauskas, E. P. Bjorntorp, M. Fahlen, I. Prerovsky, and J. Stenberg. 1970. Effects of physical training on exercise blood flow and enzymatic activity in skeletal muscle. Cardiovasc. Res. 4:418-422.

27. Mackie, B. G., and R. L. Terjung. 1983. Influence of training on blood flow to different skeletal muscle fiber types. J. Appl. Physiol. 55:1072-1078.

28. Sale, D. 1987. Influence of exercise and training on motor unit activation. Exercise Sport Sci. Rev. 15:95-151.

29. Moritani, T., and H. A. DeVries. 1979. Neural factors versus hypertrophy in the time course of muscle strength gain. Am. J. Physiol. Med. 58:115-130.

30. Dudley, G. A., P. C. Tullson, and R. L. Terjung. 1987. Influence of mitochondrial content on the sensitivity of respiratory control. J. Biol. Chem. 262:9109-9114.

31. Chance, B., J. S. Leigh, Jr., B. J. Clark, J. Maris, J. Kent, S. Nioka, and D. Smith. 1985. Control of oxidative metabolism and oxygen delivery in human skeletal muscle: a steady-state analysis of the work/energy cost transfer function. Proc. Natl. Acad. Sci. USA. 82:8384-8388.

32. Chance, B., J. S. Leigh, Jr., J. Kent, and K. McCully. 1986. Metabolic control principles and ${ }^{31}$ P NMR. Fed. Proc. 45:2915-2920.

33. Gollnick, P. D., and B. Saltin. 1982. Significance of skeletal muscle oxidative enzyme enhancement with endurance training. Clin. Physiol. (Oxf.). 2:1-12. 